\title{
BIO.05 - Evaluation of the in vitro biological activity of biosimilar and biobetter versions of rituximab developed in Bio-Manguinhos for lymphoma treatment
}

Ana Beatriz Teixeira Frederico ${ }^{1 *}$; Maria da Glória Martins Teixeira ${ }^{1}$; Eneida Almeida Santos ${ }^{1}$; Luciana Rodrigues Carvalho Barros²; Mariana Saldanha Viegas Duarte²; Martín Hernán Bonamino $^{2}$; Aline Almeida Oliveira ${ }^{1}$.

1Fiocruz/Bio-Manguinhos;

2INCa.

Introduction: Monoclonal antibodies (mAbs) have revolutionized cancer treatment since the approval of Rituximab for non-Hodgkin's lymphoma (NHL). New anti-CD20 antibodies have been developed, including biosimilars and biobetters. Biosimilars are mAbs that have the same amino acid sequence as the reference $\mathrm{mAb}$ (commercial therapeutic product) but are produced by other manufacturing processes. Biobetters have the same target epitope as the reference $\mathrm{mAb}$, however they do have some structural modifications to make it better than the reference mAb. Since many patients do not respond to treatment with anti-CD20 antibodies, biobetters appear as a valid alternative for the treatment of NHL. The main mode of action of these anti CD20 mAbs is through Antibody-Dependent Cellular Citotoxicity (ADCC), involving the activation of Natural Killers (NK) cells. These cells can act through the interaction of receptors such as NKG2D with its ligands (NKG2DL) found on the surface of target cells. Our group proposes the development of a biosimilar of rituximab and the creation of a biobetter antibodies based on the addition of an NKG2D ligand to the $\mathrm{mAb}$ structure.

Objective: This work aims to evaluate the biosimilar and biobetters of rituximab developed in BioManguinhos regarding their ability to bind to the CD20 antigen and the in vitro biological activity.

Methodology: Through flow cytometry assays it was possible to analyze the binding properties of the constructs to the CD20 antigen on the surface of leukemia cells (K562 CD20+) and the presence of NKG2DL in the constructs. Potential ADCC and CDC were evaluated using the CytoTox96 kit, a non-radioactive cytotoxicity colorimetric assay capable of measuring the lactate dehydrogenase in the medium, and NK cells expanded in vitro as effector cells.

Results: Flow cytometry assays have demonstrated that the constructs produced in BioManguinhos are capable of binding to CD20+ cells and that NKG2DLs are present in the biobetters constructs. ADCC and CDC assays demonstrated that the presence of NKG2DLs in the constructs improved the in vitro biological activity of the $\mathrm{mAb}$, evidencing a higher percentage of cell lysis of the biobetter when compared to the biosimilar and rituximab (MabThera) antibodies.

Conclusion: The conclusion is that the antibodies developed are capable of binding to CD20 expressing cells and the biobetters presented improved biological activity in vitro when compared to the biosimilar and reference rituximab, suggesting that the addition of the NK-cell ligand to the antiCD20 mAb may enhance the therapeutic efficacy of rituximab and other therapeutic antibodies. To reinforce this hypothesis, we have established an in vivo model consisting in xenografts of CD20+ the human lymphoma cell line (RAJI) in immunodeficient mice. The immunodeficient animals will be treated with primary human NK cells and/or the mAbs of interest to evaluate the in vivo enhancing function of the new conformations of the anti CD20 mAbs.

Keywords: Monoclonal antibody; Anti-CD20; Biological activity 